\title{
Acknowledgement to Reviewers of Robotics in 2019
}

\author{
Robotics Editorial Office
}

MDPI, St. Alban-Anlage 66, 4052 Basel, Switzerland

Published: 27 January 2020

The editorial team greatly appreciates the reviewers who have dedicated their considerable time and expertise to the journal's rigorous editorial process over the past 12 months, regardless of whether the papers are finally published or not. In 2019, a total of 103 papers were published in the journal, with a median time to first decision of 27 days and a median time from submission to publication of 52 days. The editors would like to express their sincere gratitude to the following reviewers for their generous contribution in 2019:

Abe, Naoko

Adamatzky, Andrew

Aghasian, Erfan

Ahmed, Sevil

Alsalloum, Ataa

Alves De Oliveira, Thiago Eustaquio

Auat-Cheein, Fernando

Bai, Long

Barrett, Ronald M.

Bellotto, Nicola

Berg, Devin R.

Bianchi, Matteo

Bimbo, Joao

Boboc, Răzvan Gabriel

Bocicor, Maria Iuliana

Bonaci, Tamara

Boscariol, Paolo

Boschetti, Giovanni

Bouffanais, Roland

Bozcuoğlu, Asil Kaan

Brito-Loeza, Carlos

Calderon, Juan

Calli, Berk

Cao, Hoang-Long

Carbone, Giuseppe

Castillo Garcia, Fernando Jose

Castillo, Oscar

Chablat, Damien

Chamoso Santos, Pablo

Chang, Bo

Chang, Chung-Liang

Chen, Dechao

Cheng, Shing Shin

Cheng, Yongqiang

Cho, Dang-il
Choi, Jaesoon

Chou, Chien-Hsing

Clark, Jason

Cocuzza, Silvio

Coevoet, Eulalie

Conti, Daniela

Controzzi, Marco

Cooper, Matthew A.

Corrales Ramon, Juan Antonio

Corves, Burkhard

Costa Ramos, Daniel

Cristófol-Rodríguez, Francisco-Javier

Cronkite, Ruth C.

Da Costa, Cesar

D'Alonzo, Marco

De Oliveira, Mario Anderson

De Rossi, Danilo

Deshpande, Nikhil

Deussen, Oliver

Di Natali, Christian

Di Nuovo, Alessandro

Dias, Andre

Doitsidis, Lefteris

Dos Santos, Matheus

Droge, Greg

Efthimiou, Eleni

Eteokleous, Nikleia

Fakhari, Amin

Fan, Jizhou

Fekrmandi, Hadi

Feliu, Vicente

Fernandes, Marcelo

Ferraresi, Carlo

Foit, Krzysztof

Fomin, Alexey 
Forner-Cordero, Arturo

Fortuna, Luigi

Fourlas, George K.

$\mathrm{Fu}$, Hailing

Fujita, Katsuhide

Gallardo-Alvarado, Jaime

Gao, Fei

Gao, Feng

Garcia Gonçalves, Luiz Marcos

Gasparetto, Alessandro

Gawel, Abel

Giberti, Hermes

Giffney, Tim

Giorgio-Serchi, Francesco

Girbés, Vicent

Gonzalez De Los Reyes, Rafael Corsino

Gonzalvez, Pablo Rodriguez

Goodin, Christopher T.

Grazioso, Stanislao

Grosch, Patrick

Groves, Keir

Gruppioni, Emanuele

Guan, Yisheng

Guo, Shuxiang

Hariri, Hassan Hussein

Haschke, Robert

$\mathrm{He}$, Qingsong

Hernandez, Eusebio Eduardo

Herrera-May, Agustin L.

Hintze, Wolfgang

Hockman, Benjamin

Hohmann, Sören

Holm, Magnus

Holthaus, Patrick

Horla, Dariusz

Huang, Sunan

Hussien, Haitham

Indri, Marina

Ioannis, Liritzis

Ivanescu, Mircea

James, Jasper

Jerčić, Petar

Jovanović, Vukica

Jung, Sunghun

Kaboli, Mohsen

Kamel, Mohamed A.

Kapadia, Apoorva

Karaszewski, Maciej

Kawashima, Kenji

Kazanzides, Peter

Kelemen, Michal

Kermani, Mehrdad R.
Kim, Jong-Hoon

Kosa, Gabor

Kostavelis, Ioannis

Kouziokas, Georgios N.

Kožuh, Ines

Krajnik, Tomas

Kumon, Makoto

Kurabayashi, Daisuke

Labbé, Mathieu

Lara Molina, Fabian Andres

Laribi, Med Amine

Laski, Pawel Andrzej

Lee, Hyun-Taek

Lee, Min

Lennox, Barry

Li, Caihong

Li, Chi

Li, Miao

Liarokapis, Minas

Liu, Guangjun

Liu, Guoliang

Liu, Huaping

Liu, Tingyi

López, Antonio M.

Loria, Antonio

Lovasz, Erwin-Christian

Lozenguez, Guillaume

Luo, Cai

Ma, Ji

Ma, Raymond

Magnusson, Martin

Mahajan, Harshal

Makris, Sotiris

Malvezzi, Monica

Manfredi, Luigi

Mangina, Eleni

Martinez-Carranza, José

Martinez-Martin, Ester

Martín-Rico, Francisco

Martins Da Silva, Maira

Mateo, Carlos M.

Mazomenos, Evangelos

McGrath, James

Mejia Rincon, Leonardo

Meng, Lingju

Mercorelli, Paolo

Mizzi, Luke

Monaco, Vito

Mondada, Francesco

Moore, Philip

Moreno-Valenzuela, Javier

Mostafa, Mostafa 
Motzki, Paul

Mura, Andrea

Muscato, Giovanni

Mutka, Alan

Naceri, Abdeldjallil

Nansai, Shunsuke

Napolitano, Rebecca

Nascimento, Tiago

Natale, Ciro

Neveln, Izaak

Nguyen, Thanh Thi

Nikolaidis, Stefanos

Novak, Domen

Oswald-Tranta, Beate

Otani, Takuya

Palacín, Jordi

Palinko, Oskar

Pandya, Abhilash

Pang, Da-Chen

Park, Jeonghong

Parmiggiani, Alberto

Parra, Vicente

Patel, Sarosh

Patil, Madhav

Pellenz, Johannes

Perez-Ramirez, Carlos

Perikos, Isidoros

Pertile, Marco

Peterson, John

Petit, Antoine

Pires, Ivan

Pirozzi, Salvatore

Pollicino, Antonino

Popescu, Diana

Pranggono, Bernardi

Primatesta, Stefano

Pytka, Jarosław

Rabindran, Dinesh

Rahman, M Arifur

Rajasekaran, Vijaykumar

Ramon Soria, Pablo

Rangel-Magdaleno, José De Jesús

Rasolofondraibe, Lanto

Ravankar, Abhijeet

Realmuto, Jonathan

Reinoso, Oscar

Rodríguez-Licea, Martín-A.

Roldán Gómez, Juan Jesús

Romero, Margarida

Roth, Zvi

Roveda, Loris

Rybus, Tomasz
Rzucidlo, Pawel

Saal, Hannes

Sabatini, Marco

Šafarič, Riko

Saltaren, Roque

Salzman, Oren

Sanchez-Lopez, Jose Luis

Sattar, Junaed

Sauer, Piotr

Savino, Heitor

Sawada, Tadamasa

Scalera, Lorenzo

Scaradozzi, David

Screpanti, Laura

Seet, Gerald Gim Lee

Seriani, Stefano

Severson, Rachel

Shih, Ching-long

Siguerdidjane, Houria

Simoni, Roberto

Singh, Aditi

Song, Rong

Song, $\mathrm{Yu}$

Sparavigna, Amelia Carolina

Stilwell, Daniel

Straub, Jeremy

Strisciuglio, Nicola

Stuart, Hannah

$\mathrm{Su}$, Junwei

Šuligoj, Filip

Sun, Yanchao

Sutin, Alexander

Takeda, Yukio

Tanev, Ivan

Tibaduiza Burgos, Diego Alexander

Tiseo, Carlo

Tkach, Itshak

Torres-Méndez, Luz Abril

Trematerra, Amelia

Trovato, Gabriele

Tsiakas, Konstantinos

Tuyishimire, Emmanuel

Tyrrell, Andy

Umbrico, Alessandro

Vaida, Calin

Valiente, David

Valova, Irena

Valverde, Alfredo

Vander Hook, Joshua

Vieira Filho, Jozue

Visentin, Francesco

Vladareanu, Luige 
Vonásek, Vojtěch

Wang, Jiangxin

Wang, Miaomiao

Wang, Yue

Watson, Simon

Wehrle, Erich

Wehrmeister, Marco

Wilke, Daniel

Woo, Jinseok

Wood, Stephen L.

$\mathrm{Wu}$, Huapeng

Xiang, Chaoqun

Xiang, Xianbo

Xiao, Yong

$\mathrm{Xu}$, Hongli

$\mathrm{Xu}$, Wenjun

Yamaguchi, Tomoyuki

Yang, Guanci

Yang, Yang

Yao, Xifan
Yazdani, Mojtaba

Yi, Daqing

Yu, Junzhi

Yu, Kaiyan

Yue, Tao

Zahadat, Payam

Zaplana, Isiah

Zhang, Haichong

Zhang, Heye

Zhang, Hong

Zhang, Hongying

Zhang, Jian

Zhang, Wenzeng

Zhang, Xiaoyong

Zhang, Zhou

Zhou, Dalin

$\mathrm{Zi}$, Bin

Zubizarreta, Asier

Zubrycki, Igor

(C) 2020 by the author. Licensee MDPI, Basel, Switzerland. This article is an open access article distributed under the terms and conditions of the Creative Commons Attribution (CC BY) license (http://creativecommons.org/licenses/by/4.0/). 